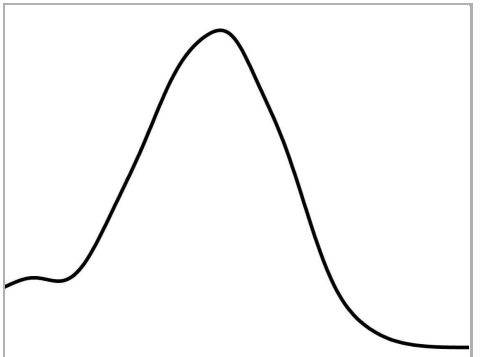

$\begin{array}{llllllllll}20 & 30 & 40 & 50 & 60 & 70 & 80 & 90\end{array}$ Speed $(\mu \mathrm{m} / \mathrm{s})$

\section{MAR 02, 2021}

\section{open ठaccess}

DOI:

dx.doi.org/10.17504/protocol s.io.bsw5nfg6

Protocol Citation: Joao Vitor Molino 2021. Chlamydomonas reinhardtii cell motility quantification. protocols.io https://dx.doi.org/10.17504/p rotocols. io.bsw 5 nfg 6

License: This is an open access protocol distributed under the terms of the Creative Commons Attribution License, which permits unrestricted use, distribution, and reproduction in any medium, provided the original author and source are credited

Protocol status: Working We use this protocol and it's working

Created: Mar 02, 2021

Last Modified: Mar 02, 2021

PROTOCOL integer ID:

47805

\section{(3) Chlamydomonas reinhardtii cell motility quantification}

\author{
Joao Vitor Molino ${ }^{1}$
}

${ }^{1}$ Ronin Institute

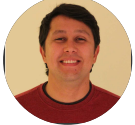

Joao Vitor Molino

Ronin Institute

\section{DISCLAIMER}

\author{
DISCLAIMER - FOR INFORMATIONAL PURPOSES ONLY; USE AT YOUR OWN \\ RISK
}

The protocol content here is for informational purposes only and does not constitute legal, medical, clinical, or safety advice, or otherwise; content added to protocols.io is not peer reviewed and may not have undergone a formal approval of any kind. Information presented in this protocol should not substitute for independent professional judgment, advice, diagnosis, or treatment. Any action you take or refrain from taking using or relying upon the information presented here is strictly at your own risk. You agree that neither the Company nor any of the authors, contributors, administrators, or anyone else associated with protocols.io, can be held responsible for your use of the information contained in or linked to this protocol or any of our Sites/Apps and Services.

\section{ABSTRACT}

This protocols describe the steps required for the motility quantification in Chlamydomonas reinhardtii.

\section{GUIDELINES}

All steps described in this protocol are intended to be conducted in a research laboratory. Follow aseptic procedures.

\title{
Cell preparation
}

1 1. Culture the cells for 5 days following the growing Chlamydomonas reinhardtiiprotocol.

2. Prepare a sample of the cells by washing them in the final desired media by centrifugation. 


\section{Glass slide preparation}

2 1. In glass slide, attach the Frame-seal Slide chambers, $15 \times 15,65 \mu \mathrm{l}$.

2. Add the sample

3. Seal the chamber with a cover slip

\section{Imaging}

3 1. Set the glass slide in the microscope for image acquisition

2. Record sequential images in a fixed interval (e.g. $100 \mathrm{~ms}$ per frame) using the desired laser/filter settings.

\section{Cell tracking analysis - imageJ}

4 1. Open the image file in image $J$

2. Adjust brightness and contrast (Image -> Adjust -> Brightness/Contrast -> auto)

3. Open the Plugins -> Tracking -> TrackMate

4. Check the image settings (eg. Size and pixels), next

5. Choose the detector system, next

6. Input the estimate size of cells and define a threshold, next

7. After detection, choose a range of spots quality, next

8. Select a view option, next

9. Set filter in spots, next

10. Define the maximum linking distance between frames, next

11. Set color for tracks, next

12. Open analysis and save the results in a csv file

*The analysis files contains information about the tracks, as average speed, max speed, position, etc.

** A video demonstrating the TrackMate workflow can be found here.

\section{Data analysis}

5 Analyse the data generated. For example, after organizing the data of each test in a excel tab, the $\mathrm{R}$ code below demonstrate a possible workflow and analysis to generate a density plot.

library (dplyr)

library (ggplot2) 


\section{library ( readxl)}

\#set the working directory

wd <- setwd("D:/Path")

\#Get all file names with. xlsx in the working folder.

FileNames <- Sys.glob("*.xlsx")

\#load file with results

cc1690_1 <- read_excel("UZH20201206 Motility.xlsx", sheet = "1690_1")

cc1690_2 <- read_excel("UZH20201206 Motility.xlsx", sheet = "1690_2")

cc1690_3 <- read_excel("UZH20201206 Motility.xlsx", sheet = "1690_3")

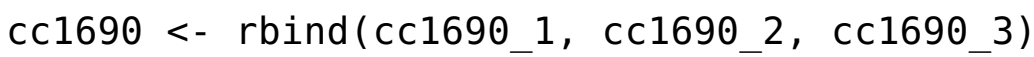

MAW8_1 <- read_excel("UZH20201206 Motility.xlsx", sheet = "MAW8_1")

MAW8_2 <- read_excel("UZH20201206 Motility.xlsx", sheet = "MAW8_2")

MAW8_3 <- read_excel("UZH20201206 Motility.xlsx", sheet = "MAW8_3")

MAW8 <- rbind(MAW8_1, MAW8_2, MAW8_3)

GP1_1 <- read_excel("UZH20201206 Motility.xlsx", sheet = "GP1_1")

GP1_2 <- read_excel("UZH20201206 Motility.xlsx", sheet = "GP1_2")

GP1_3 <- read_excel("UZH20201206 Motility.xlsx", sheet = "GP1_3")

GP1 <- rbind $\left(\mathrm{GP} 1 \_1, \mathrm{GP} 1 \_2, \mathrm{GP1} 3\right)$

\#Take the last 10 seconds of the experiment

cc1690 <- filter (cc1690, start_t > 50)

MAW8 <- filter(MAW8, start_t $>50$ )

GP1 <- filter(GP1, start_t > 50) 
\#Add column with Strain information

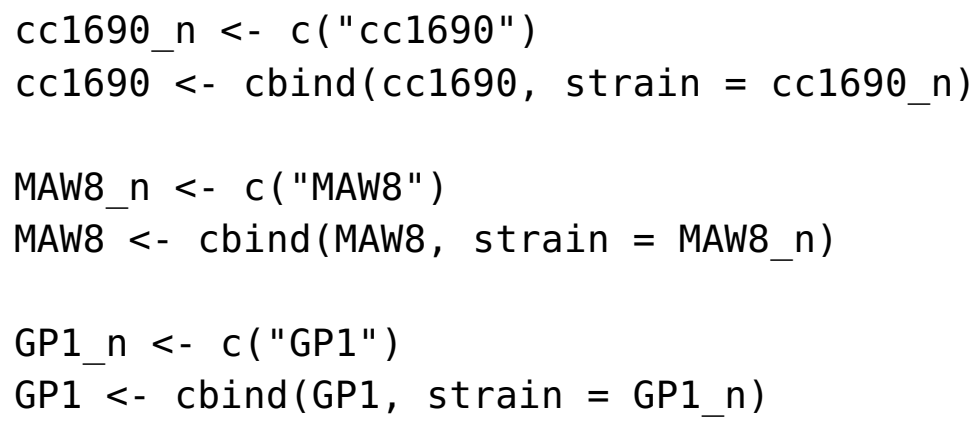

\#Combine data

df <- rbind (cc1690, MAW8, GP1)

\# Convert data to speed um/s (File recorder at 100ms/frame|speed in frames/s)

df\$speed_r $<-$ df $\$$ speed*10

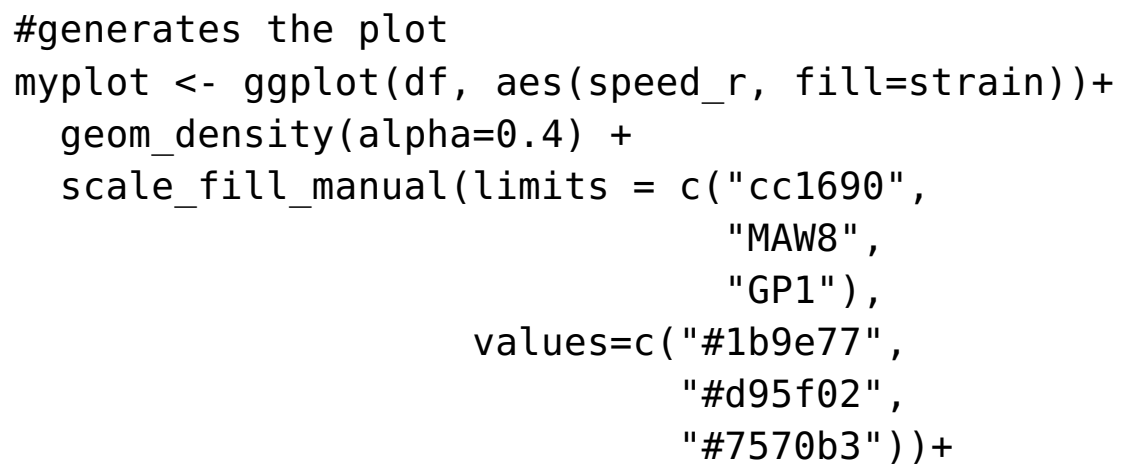


